Thorax (1973), 28, 113.

\title{
An intragastric cardiac pacemaker
}

\author{
H. A. SAMAAN \\ Department of Thoracic Surgery, The Royal Infirmary, Edinburgh
}

This paper describes a functioning cardiac pacemaker which was found to be inside the stomach, although originally placed within the rectus sheath.

\section{CASE REPORT}

A 62-year-old housewife was seen in May 1967 with attacks of dizziness and loss of consciousness. A diagnosis of complete heart block with Stokes-Adams syncope was made. Anterolateral thoracotomy was performed and myocardial electrodes were inserted over the left ventricle and were connected to a Devices fixed-rate pacemaker, inserted in the rectus sheath posterior to the rectus muscle. Postoperatively the pacemaker functioned satisfactorily at a rate of 72 per minute. The patient remained well and the pacemaker was replaced by an experienced sur-

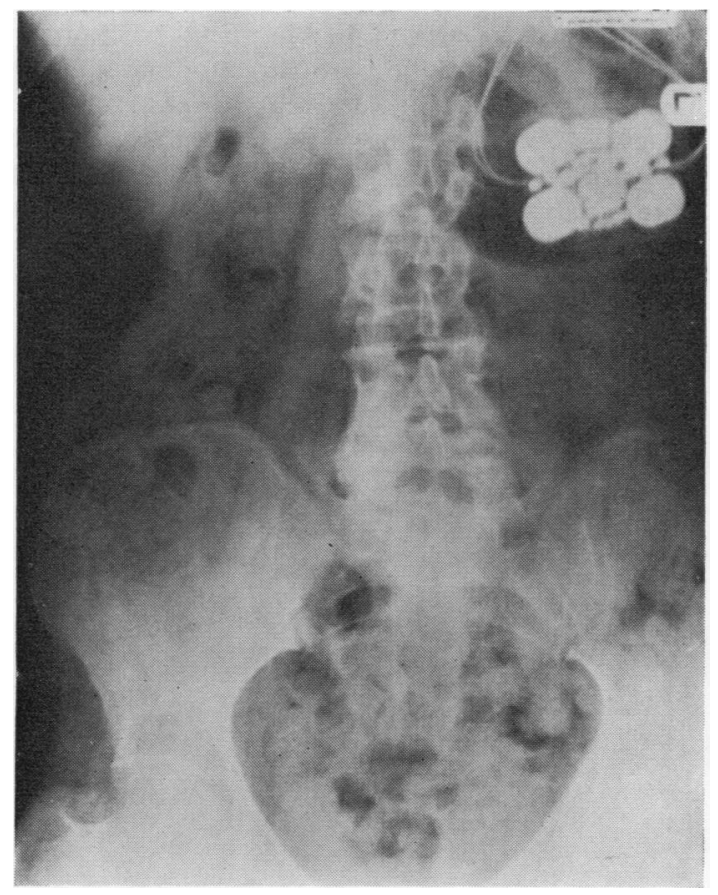

FIG. 1. Plain radiograph of abdomen showing the position of the pacemaker before removal. geon, using the same pocket, in July 1968 and in January 1970 , because of gradual failure of its voltage output. The patient attended every three months for a check of pacemaker activity.

In September 1971, the output started to fail. A radiograph of the abdomen is shown (Fig. 1). The pacemaker was considered to be in the anterior abdominal wall. Under general anaesthesia the previous abdominal scar was excised but the pacemaker was not palpable in the upper abdominal wall. The peritoneum was incised and the pacemaker was felt lying freely mobile within the cavity of the stomach.

The stomach was incised and the pacemaker was found to be lying free in the gastric cavity (Fig. 2). The silicone rubber did not seem to be digested. The cardiac electrodes passed through the stomach to the diaphragm and the left ventricle, while the indifferent electrodes were embedded in the inner surface of the stomach. The pacemaker was removed and the stomach wall was sutured. When tested, the cardiac ends of the electrodes were found to be unsatisfactory owing to the high voltage required for cardiac pacemaking. A temporary endocardiac pacemaker using the right subclavian vein was inserted. The abdominal wall was closed. A few days later, a permanent endocardial pacemaker using the left cephalic vein with a pulse generator in the axilla was inserted. The patient made a satisfactory recovery.

\section{DISCUSSION}

It is difficult to explain the presence of the pacemaker within the gastric lumen. There are only two alternatives - either it was originally placed in the stomach or it migrated there from the rectus sheath. There was no record that the stomach was opened at any previous operation. There was also no evidence of previous peritonitis. However, the patient remained well for 21 months and never complained of any digestive disturbance and was unaware of any foreign body in the stomach. Owing to the attachment of the indifferent electrode to the stomach it is possible 


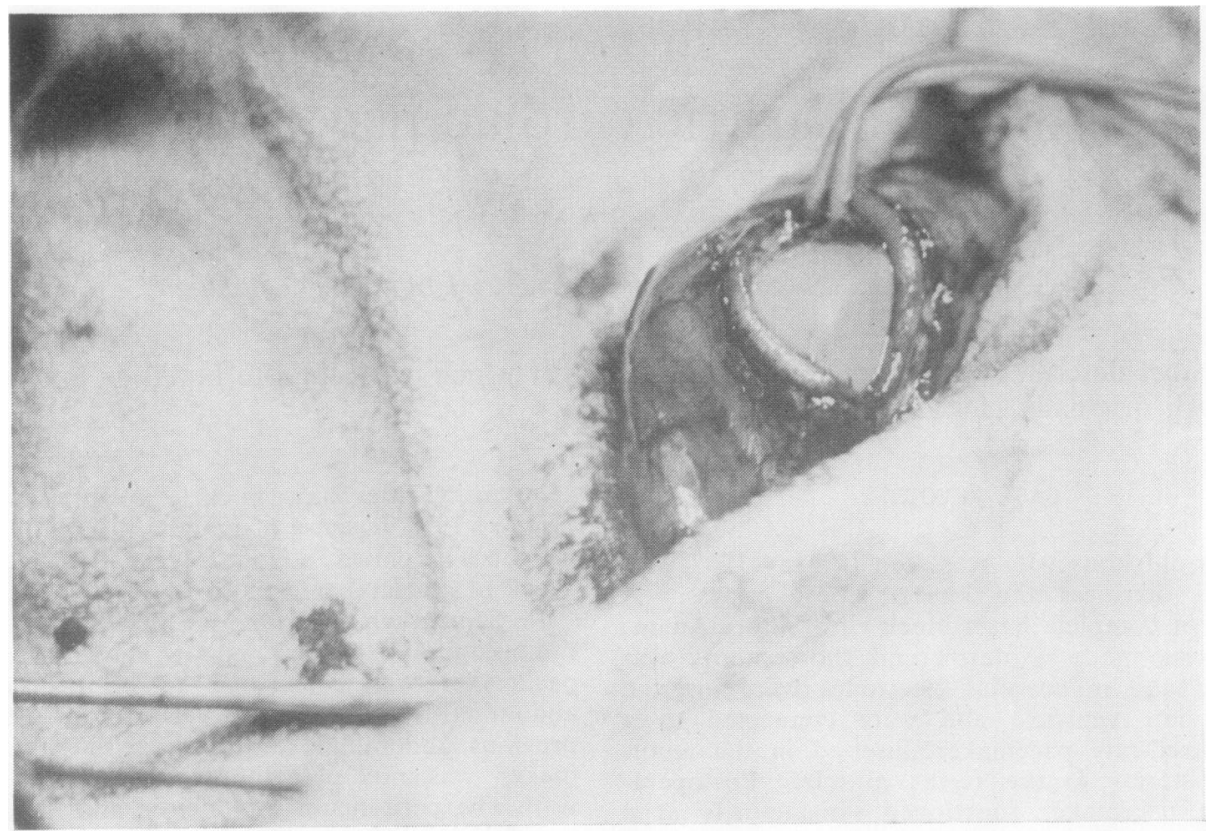

FIG. 2. The pacemaker inside the lumen of the stomach.

that the stomach was electrically stimulated each time the heart contracted.

The pacemaker was covered by silicone rubber and functioned satisfactorily throughout.

It is possible that the pacemaker may not have been inside the stomach for the period of 21 months. Pacemakers inserted within or behind the rectus sheath tend to change their position $b \overrightarrow{\bar{\Phi}}$ gravity and contraction of surrounding muscles. A thin fibrous capsule derived from surrounding tissues usually surrounds and envelops the pacemaker. In this case, no fibrous capsule was present around the pacemaker when it was removed from inside the stomach. 